\title{
Intraoperative very late stent thrombosis in patient undergoing arthroscopic shoulder surgery
}

\author{
In Young Huh, Hee Won Son, An Suk Kim, Minha Sung, and Soon Eun Park \\ Department of Anesthesiology and Pain Medicine, Ulsan University Hospital, Ulsan, Korea
}

One of the therapeutic strategies for patients with coronary artery disease (CAD) is coronary stenting. Stent implantation induces endothelial damage, which leads to neointimal hyperplasia and increases the risk of thrombosis [1]. Drug-eluting stents (DESs), in which various drug coatings have been applied to the stents, are aimed at reducing neointimal hyperplasia and restenosis [1]. A rare, but catastrophic complication from DES implantation is stent thrombosis, which can lead to myocardial infarction (MI) and sudden death [1]. Clopidogrel and aspirin are crucial for stent thrombosis prevention.

In the following case, a patient with a history of DES implantation 5 years prior suddenly developed unexpected circulatory collapse and ventricular fibrillation during arthroscopic shoulder surgery.

A 62 year-old man $(72 \mathrm{~kg}$, American Society of Anesthesiologists physical status II) was scheduled to receive elective operation for an arthroscopic shoulder. He had experienced two incidences of CAD (5 years and 4 years ago), following which he had received a DES implant $\left(\right.$ CYPHER $^{\circledR}$, Cordis Corp., Bridgewater, NJ, USA) in the left distal main to left anterior descending artery (LAD) and ballooning in the left main to proximal left circumflex artery (pLCX) and LAD, respectively. Aspirin and clopidogrel were initiated following the stent procedure, but were stopped by his cardiologist 5 days before the elective operation. The patient underwent an echocardiography, and his left ventricular function was reported as normal for his age.

After arterial catheterization was performed at the left radial artery, the patient's blood pressure (BP) was 152/95 $\mathrm{mmHg}$ and his heart rate (HR) was 79 beats per minute (bpm). After anes- thesia was induced, the desflurane and remifentanil were adjusted according to BP and bispectral index. The patient was placed in the beach chair position. The BP monitoring transducer was placed at the external auditory canal to monitor the cerebral perfusion pressure, due to compromised cerebral perfusion. Although the surgeon sought a hypotensive condition in order to achieve bloodless field, we tried to maintain the mean BP in the region of $60 \mathrm{mmHg}$, considering the patient's cardiac status. The BP decreased to $75 / 38 \mathrm{mmHg}$ and was restored to $100 / 70$ $\mathrm{mmHg}$, following an injection of ephedrine $10 \mathrm{mg}$ and phenylnephrine $100 \mu \mathrm{g}$. The surgery was lasted 104 minutes and was uneventful, with no change in ECG (BP 90-100/40-45 mmHg, HR 100-105 bpm). The BP suddenly dropped to $73 / 30 \mathrm{mmHg}$ at the end of the surgery. The patient was placed in the supine position, and norepinephrine was administered twice (20 $\mu \mathrm{g}$ each time) and then continuously infused. However, the BP did not recover. Subsequently, ventricular fibrillation began to manifest and cardiopulmonary resuscitation was performed. The arterial blood gas analysis (ABGA) at $\mathrm{FIO}_{2} 1.0$ was $\mathrm{pH}$ 7.27, $\mathrm{PCO}_{2}$ $37 \mathrm{mmHg}, \mathrm{PO}_{2} 49 \mathrm{mmHg}$, and bicarbonate $15.6 \mathrm{mEq} / \mathrm{L}$. As the vital signs did not improve, extracorporeal membrane oxygenation (ECMO) was applied via the right femoral artery and vein by the cardiothoracic surgeon. The ECMO flow was maintained at $0.8-1.2 \mathrm{~L} / \mathrm{min}$, and the $\mathrm{BP}$ was $60-75 / 30-42 \mathrm{mmHg}$. ABGA

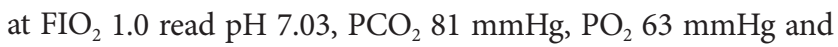
bicarbonate $21.4 \mathrm{mEq} / \mathrm{L}$. We attempted to increase the ECMO flow with volume replacement, but this proved difficult.

Very late stent thrombosis was suspected. A thrombectomy and successful stenting of the left main to LAD and pLCX (T-

Corresponding author: In Young Huh, M.D., Department of Anesthesiology and Pain Medicine, Ulsan University Hospital, 877, Bangeojinsunhwando-ro, Dong-gu, Ulsan 682-714, Korea. Tel: 82-52-250-7246, Fax: 82-52-250-7249, E-mail: inyoung_huh@uuh.ulsan.kr

(c) This is an open-access article distributed under the terms of the Creative Commons Attribution Non-Commercial License (http:// creativecommons.org/licenses/by-nc/3.0/), which permits unrestricted non-commercial use, distribution, and reproduction in any medium, provided the original work is properly cited. 
stenting) were therefore performed. The echocardiography revealed a severe left ventricular systolic dysfunction and ischemic insult of the LAD and LCX territory. In the intensive care unit, the vital sign showed BP 91/75 mmHg, HR 121 bpm and body temperature $33^{\circ} \mathrm{C}$, due to the induced therapeutic hypothermia. On the postoperative day (POD) 1 , the vital signs read BP 90/55 mmHg, HR $110 \mathrm{bpm}$ and temperature $33^{\circ} \mathrm{C}$. On POD 2, the vital signs and ABGA had deteriorated (BP 70/45 $\mathrm{mmHg}$, HR 117 bpm, ABGA pH 7.13, $\mathrm{PCO}_{2} 83$ mmHg, $\mathrm{PO}_{2} 60 \mathrm{mmHg}$, bicarbonate $27 \mathrm{mEq} / \mathrm{L}$, base excess $-3.3, \mathrm{SaO}_{2} 81 \%$ ). With the aggravating occurrences of hypotension, hypoxemia, and sustained ventricular fibrillation arrest, the patient could not be resuscitated.

This case presented an instance of very late stent thrombosis, which might have been related to the interruption of the dual antiplatelet therapy during the perioperative period. The risk factors for perioperative late stent thrombosis with DES could be: stents implanted in the left main coronary artery or in bifurcations, restenosis, increased stent length, several numbers of stents, patient related factors which may delay wound healing, and inappropriate discontinuation of the dual antiplatelet therapy [2]. A prothrombotic state and inflammatory activation induced by the surgery can also increase the risk of perioperative stent thrombosis [3]. Aspirin withdrawal causes an increase in cyclooxygenase- 1 and thromboxane $\mathrm{B} 2$, producing thrombin and decreasing fibrinolysis, which further enhances the platelet aggregation, and consequently increases the risk of stent throm- bosis [4]. Aspirin withdrawal in patients with CAD is a strong independent predictor of MI, especially in patients with a coronary stent [5]. Thus, the perioperative discontinuation of aspirin is only recommended when the risk of bleeding complications exceeds the cardiovascular risk from aspirin withdrawal in patients with a risk of stent thrombosis. In our case, the patient presented several risk factors for very late stent thrombosis: a bifurcation lesion involving the left main coronary artery, a history of re-stenosis, and preoperative interruption of the dual antiplatelet therapy.

Furthermore, tachycardia and the induced hypotension to reduce the bleeding in the operation field, related to decrease coronary perfusion, are possible causes of this event. The beachchair position and induced hypotension for shoulder surgery present potential risks of systemic hypoperfusion, and can be causes of adverse outcomes, such as MI and strokes. The BP should be maintained close to the pre-anesthetic values in order to preserve the organ perfusion.

When intraoperative stent thrombosis occurs, it abruptly causes as MI or malignant dysrhythmia, and should therefore be detected and treated early, through immediate reperfusion of the coronary blood flow.

This case reveals that patients with DES are not free from risk of the very late stent thrombosis, even after completion of their dual antiplatelet therapy. We should communicate closely with other clinicians regarding patients with DES.

\section{References}

1. Iakovou I, Schmidt T, Bonizzoni E, Ge L, Sangiorgi GM, Stankovic G, et al. Incidence, predictors, and outcome of thrombosis after successful implantation of drug-eluting stents. JAMA 2005; 293: 2126-30.

2. Brilakis ES, Banerjee S, Berger PB. Perioperative management of patients with coronary stents. J Am Coll Cardiol 2007; 49: 2145-50.

3. Mangano DT. Perioperative cardiac morbidity. Anesthesiology 1990; 72: 153-84.

4. Jimenez AH, Stubbs ME, Tofler GH, Winther K, Williams GH, Muller JE. Rapidity and duration of platelet suppression by enteric-coated aspirin in healthy young men. Am J Cardiol 1992; 69: 258-62.

5. Biondi-Zoccai GG, Lotrionte M, Agostoni P, Abbate A, Fusaro M, Burzotta F, et al. A systematic review and meta-analysis on the hazards of discontinuing or not adhering to aspirin among 50,279 patients at risk for coronary artery disease. Eur Heart J 2006; 27: $2667-74$. 